\title{
A Preliminary Study of Korean Dual-Frequency SBAS
}

\author{
Ho Yun, Deokhwa Han, Changdon Kee ${ }^{\dagger}$ \\ School of Mechanical and Aerospace Engineering, Seoul National University, Seoul 151-742, Korea
}

\begin{abstract}
A Satellite Based Augmentation System (SBAS) is a representative differential GNSS system, which is used for the navigation performance improvement of Global Navigation Satellite System (GNSS) users. SBAS has been developed focusing on the securement of user integrity so that it can be used for the navigation in aviation fields. Accordingly, the development of SBAS has been completed, and it has been actively used in the United States, Europe, and Japan. As the new satellite of Global Positioning System (GPS) recently started to broadcast new civil signals (L5 frequency), the methods for improving user navigation performance in SBAS using this signal have also been studied. In Korea, to keep pace with these circumstances, full-scale SBAS development is expected to start in 2014, and studies on dual-frequency SBAS using L1/L5 frequencies will also be performed. In this study, before the full-scale development of dual-frequency SBAS in Korea, a simulation was performed to predict the performance and analyze the expected effects.
\end{abstract}

Keywords: SBAS, dual-frequency, WADGPS

\section{INTRODUCTION}

With the modernization of Global Positioning System (GPS), all the GPS satellites are expected to broadcast L5 frequency signals, which are new civil signals, as well as L1 frequency signals in the near future; and GLONASS from Russia, Galileo from Europe, and Beidou from China are also expected to broadcast dual-frequency civil signals. Accordingly, for the existing Satellite Based Augmentation System (SBAS), which has provided the enhancement information only for existing GPS L1 frequency signals, development is in progress so that it can provide services to dual-frequency and multi-constellation Global Navigation Satellite System (GNSS) users; and the internal standards for this are expected to be published around 2014. In Korea, full-scale SBAS development is expected to start in 2014, and studies on dual-frequency SBAS are expected to start in 2016. In this study, the SBAS development plan in Korea was briefly examined, and the advantages of dual-frequency

Received Jan 16, 2014 Revised Feb. 06, 2014 Accepted Feb 17, 2014

†Corresponding Author

E-mail: kee@snu.ac.kr

Tel: +82-2-880-1912 Fax: +82-2-888-2069
SBAS were analyzed. Also, the performances of dualfrequency SBAS and multi-constellation GNSS SBAS were predicted using simulation.

\section{SBAS DEVELOPMENT PLAN IN KOREA}

The SBAS development phases in Korea broadly consist of three phases. Fig. 1 shows an SBAS R\&D schedule in Korea. Phase 1 is for evaluating the feasibility of SBAS establishment in the Korean Peninsula, and it had been conducted for three years from 2003. In Phase 1, an

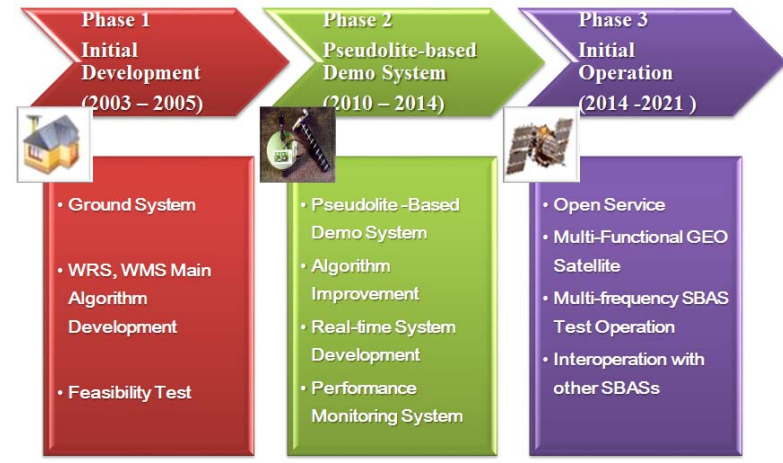

Fig. 1. SBAS R\&D schedule in Korea. 
experimental phase ground system was established using the Nation-wide Differential GPS (NDGPS) reference station infra, which had been previously established; and based on this, the main algorithms of Wide area Reference Station (WRS) and Wide area Master Station (WMS) were developed. Using the developed algorithms, the performance of SBAS, which is to be established in Korea, was predicted based on the post-processing of measured data. In Phase 2, a pseudolite-based demo system is under development by extending the system developed in Phase 1. In the Phase 1 research and development, only the main algorithms of SBAS were developed; while in Phase 2, all the algorithms excluding the part relevant to a geostationary satellite were developed. For the developed results, performance will be verified using a commercial SBAS receiver by broadcasting SBAS messages through a pseudolite. A pseudolite broadcasts SBAS messages using GPS L1 frequency signals, similar to the geostationary satellite of SBAS. Thus, a test user can evaluate the performance using equipment that is identical to an actual SBAS receiver. In the Phase 2 research and development, real-time service is available based on real-time communication with the reference station using Networked Transport of RTCM via Internet Protocol (NTRIP); and systems that play an ancillary role (e.g., performance monitoring system and database) were also developed. The Phase 3 research and development is expected to be performed from 2014, and the Korean government will officially announce the development of
SBAS. The official operation of the developed system will begin after the completion of an authentication process. Fig. 2 shows the detailed development plan of Phase 3.

For the dual-frequency SBAS which will be primarily discussed in this paper, the research and development is expected to start in 2016, and the test operation is expected from 2020.

\section{ADVANTAGE OF DUAL-FREQUENCY SBAS}

Existing SBAS provides services considering only GPS L1 frequency signal users. To provide the correction information for ionospheric delay which has the largest effect on the positioning performance of single-frequency users, existing SBAS uses a grid algorithm. In SBAS messages, the vertical ionospheric delays and their confidence levels for the grid points with latitude and longitude intervals of $5^{\circ}$ are provided across the entire service area, and users utilize these values through linear interpolation based on the ionospheric pierce point of their measurement (RTCA 2006). For ionospheric delay, deviations depending on distance and time are relatively large compared to other error factors; and especially when an ionospheric storm occurs, the deviations become larger. Therefore, a service provider has no choice but to set the confidence level conservatively in order to secure user integrity in this situation. As a result, for

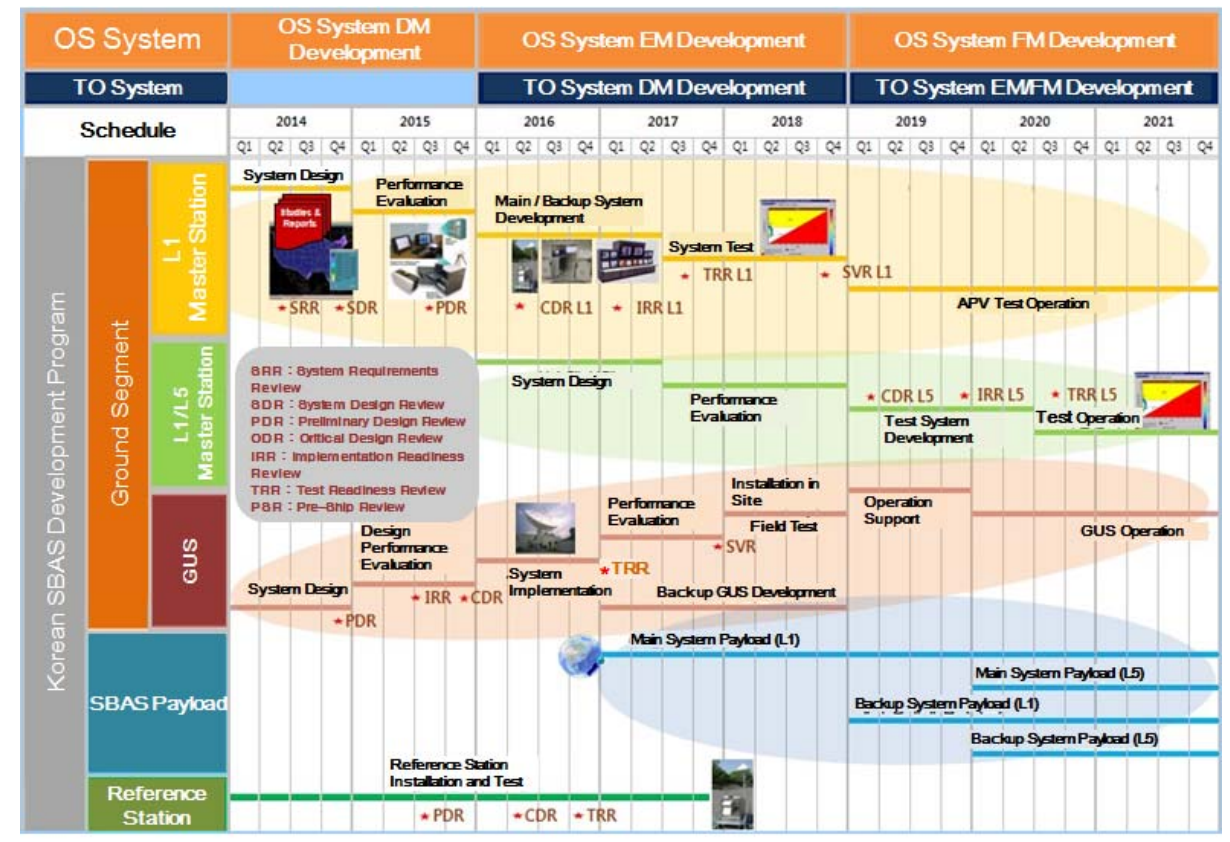

Fig. 2. SBAS development plan - phase III. 
users, the integrity of their navigation solution is secured; but in terms of availability, it cannot be improved above a certain level. However, if users utilize dual frequency, users can effectively estimate the ionospheric delay of their measurement without external aid, and thus the uncertainty of ionospheric delay estimation decreases significantly compared to single-frequency SBAS users that estimate the ionospheric delay of users based on an external system. Therefore, user protection levels decrease, and accordingly, user availability is improved (GEAS 2010). The method for estimating ionospheric delay values using L1/L5 dualfrequency measurements is explained in McGraw \& Young (2005). The measurement combination and its confidence level, which use dual-frequency measurements and are not affected by ionospheric delay, can be expressed as the following equations.

$$
\begin{gathered}
\rho_{j, D F_{-} \text {air }}=\frac{f_{1}^{2}}{f_{1}^{2}-f_{5}^{2}} \rho_{L 1, j, \text { air }}+\frac{f_{5}^{2}}{f_{1}^{2}-f_{5}^{2}} \rho_{L 5, j, \text { air }} \\
\sigma_{j, D F_{-} \text {air }}^{2}=\left(\frac{f_{1}^{2}}{f_{1}^{2}-f_{5}^{2}}\right)^{2} \sigma_{L 1, j, \text { air }}^{2}+\left(\frac{f_{5}^{2}}{f_{1}^{2}-f_{5}^{2}}\right)^{2} \sigma_{L 5, j, \text { air }}^{2}
\end{gathered}
$$

where $\rho_{j, D F_{-} \text {air }}$ represents the dual-frequency measurement combination of the $\mathrm{j}$-th satellite, and $\sigma_{j, D F_{\text {_air }}}$ represents its standard deviation. $\mathrm{f} 1$ and $\mathrm{f} 5$ represent the frequencies for L1 and L5 signals, respectively, which are 1575.42 and $1176.45 \mathrm{MHz}$, respectively. When users utilize the combination of dual-frequency measurements, ionospheric delay is eliminated via direct estimation, and thus the correction accuracy or confidence level for ionospheric delay is improved. However, in the process of combining measurements for this purpose, noise levels increase as shown in Eq. (1). Therefore, the degree of the entire error level for each case needs to be analyzed.

Table 1 summarizes the ratios for each error factor when the combination of L1/L5 frequency measurements was used and when the error of Ll frequency measurement was set as 1 (Walter et al. 2012). The nominal error affects

Table 1. L1/L5 error budget.

\begin{tabular}{lcccc}
\hline & L1 only & $\begin{array}{c}\text { Iono-free } \\
(\mathbf{L 1} / \mathbf{L 5})\end{array}$ & $\begin{array}{c}\text { Nominal } \\
\text { error }(\mathbf{m})\end{array}$ & $\begin{array}{c}\text { Max error } \\
(\mathbf{m})\end{array}$ \\
\hline Ionosphere & 1 & 0 & $0.2-1$ & 50 \\
Satellite Orbit \& Clock & 1 & 1 & $0.03-0.5$ & $\infty$ \\
Troposphere & 1 & 1 & $0.05-0.5$ & 5 \\
L1 Noise & 1 & $\frac{f_{1}^{2}}{f_{1}^{2}-f_{s}^{2}}=2.26$ & $0.15-0.5$ & 0.5 \\
L5 Noise & 0 & $\frac{f_{s}^{2}}{f_{1}^{2}-f_{s}^{2}}=1.26$ & $0.15-0.5$ & 0.5 \\
RSS & 1 & 2.6 & $0.4-1.3$ & 1.3 \\
\hline
\end{tabular}

user accuracy performance, and the max error affects user integrity and availability performance. As shown in the table, when only the L1 frequency was used, the error from ionospheric delay accounted for the largest part among the major error factors of GNSS. Especially, the max error was about $50 \mathrm{~m}$. This error could significantly deteriorate user accuracy and availability performance, and could also cause a problem for user integrity. On the other hand, if the L5 frequency signal is used together, ionospheric delay can be eliminated based on measurement combination, and thus relatively high levels of accuracy and availability could be maintained while user integrity is secured even in case of ionospheric anomaly. However, as two measurements are combined in this case, the noise level of the measurement increases as mentioned earlier. Accordingly, the overall error level of a normal state increases, and the user accuracy of a normal state could slightly deteriorate (Walter et al. 2009). Nevertheless, the accuracy performance deterioration due to the increase in the measurement noise level is insignificant, and it is expected that the accuracy for satisfying the Required Navigation Performance (RNP), which is required for the current SBAS, could be easily achieved. At present, the biggest constraint that interrupts the navigation performance improvement of L1 frequency SBAS is the reliability of ionospheric estimation. This problem could be effectively resolved using dualfrequency measurements. Therefore, it is expected that the development of dual-frequency SBAS would broaden the application fields of SBAS.

\section{DUAL-FREQUENCY SBAS SIMULATION}

To predict the performance of the dual-frequency SBAS explained earlier, a simulation for the Korean Peninsula region was carried out. The simulation time was 24 hours, and the generation methods for each error factor were presented in Table 2 (Schaer et al. 1998). To predict the minimum performance in the Korean Peninsula, the number of SBAS reference stations was set to 4 , which is the minimum number of reference stations necessary for the implementation of SBAS; and it was assumed that

Table 2. Measurement error generation for L1/L5 SBAS simulation.

\begin{tabular}{ll}
\hline \multicolumn{1}{c}{ Error factor } & Error generation method \\
\hline Satellite orbit /clock error & 2nd order Markov Process \\
Ionospheric delay error & IONEX \\
Tropospheric delay error & Black Model \\
Tropospheric delay noise & 1st order Markov Process \\
Receiver noise and multipath error & Function of Elevation Angle \\
\hline
\end{tabular}


each reference station is situated at four arbitrary locations (Gimpo, Yangyang, Mokpo, and Gimhae) that are located at the outskirts of the inland area. Users were arranged in the vicinity of the Korean Peninsula at latitude and longitude intervals of $5^{\circ}$, and the navigation performance depending on user position was analyzed. For the performance comparison of the dual-frequency SBAS, a single-frequency SBAS algorithm was also implemented; and the accuracy and availability performances when each algorithm was applied to the same error factors and measurements were analyzed. In the case of dual-frequency SBAS, the enhancement information for ionospheric delay need not be provided to users. Accordingly, the ionospheric delay correction information that accounts for about $30 \%$ of the entire message need not be broadcasted, and this part can be allotted to the correction information for other navigation system besides GPS. Thus, the establishment of dual-frequency SBAS would naturally enable the SBAS for multi-constellation GNSS. Therefore, the simulation was performed by dividing it into the case when only GPS was used and the case when GPS, GLONASS, and Galileo were used together. In the case of single-frequency SBAS, the correction information for only one navigation system can be provided at a time due to the limit of message transmission rate. However, this simulation did not place a limit on the message transmission rate, and focused only on the performance analysis of the correction information and integrity information provided from the system.

Fig. 3 shows the user accuracy performance of each system. For SBAS users, when the dual-frequency measurement was used, the accuracy performance was not different from that when only the single-frequency measurement was used; and the performance rather deteriorated slightly. This is because the measurement noise level of the normal state increased by about 2.6 times since users eliminated ionospheric delay by combining the dualfrequency measurements as explained earlier. However, as the confidence level of ionospheric delay error estimation increased, user protection levels could be estimated more accurately, and thus the protection levels decreased as shown in Fig. 4.

In Fig. 4, the GPS L1 SBAS users had an average vertical protection level of about $10 \mathrm{~m}$ only in the central part of the service area, whereas the GPS L1/L5 dual-frequency SBAS users had an average vertical protection level of about $10 \mathrm{~m}$ over the entire Korean Peninsula. The resultant increases in LPV availability are shown in Fig. 5. As shown in Figs. 3-5, when GLONASS and Galileo were added to GPS, the accuracy and availability performance increased significantly for both the single-frequency and dualfrequency SBAS. This is because the geometric arrangement of the navigation satellites was improved and the confidence level of the user navigation solution increased since the number of available measurements increased by about three times. Especially, the establishment of the dualfrequency SBAS is expected to improve the performance so that LPV service can be enabled in part of Japan and China as well as in the vicinity of the Korean Peninsula.

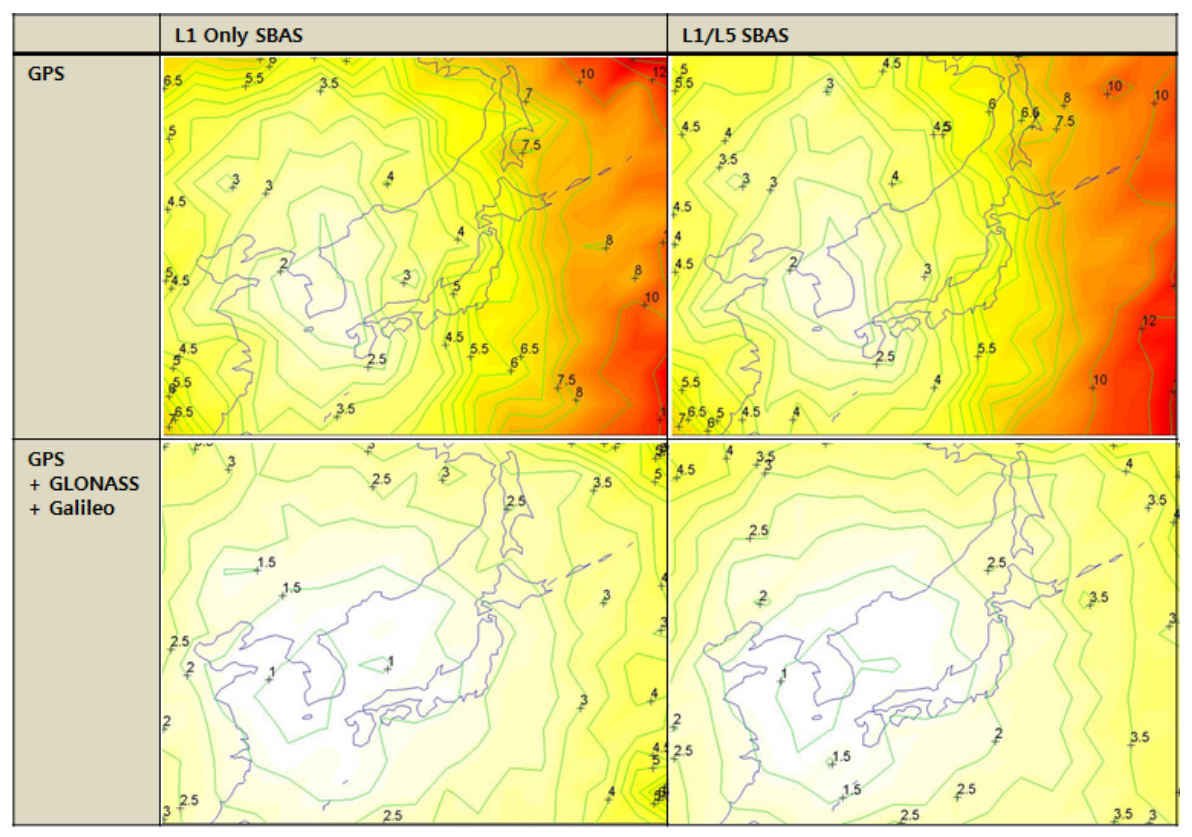

Fig. 3. User accuracy performance. 


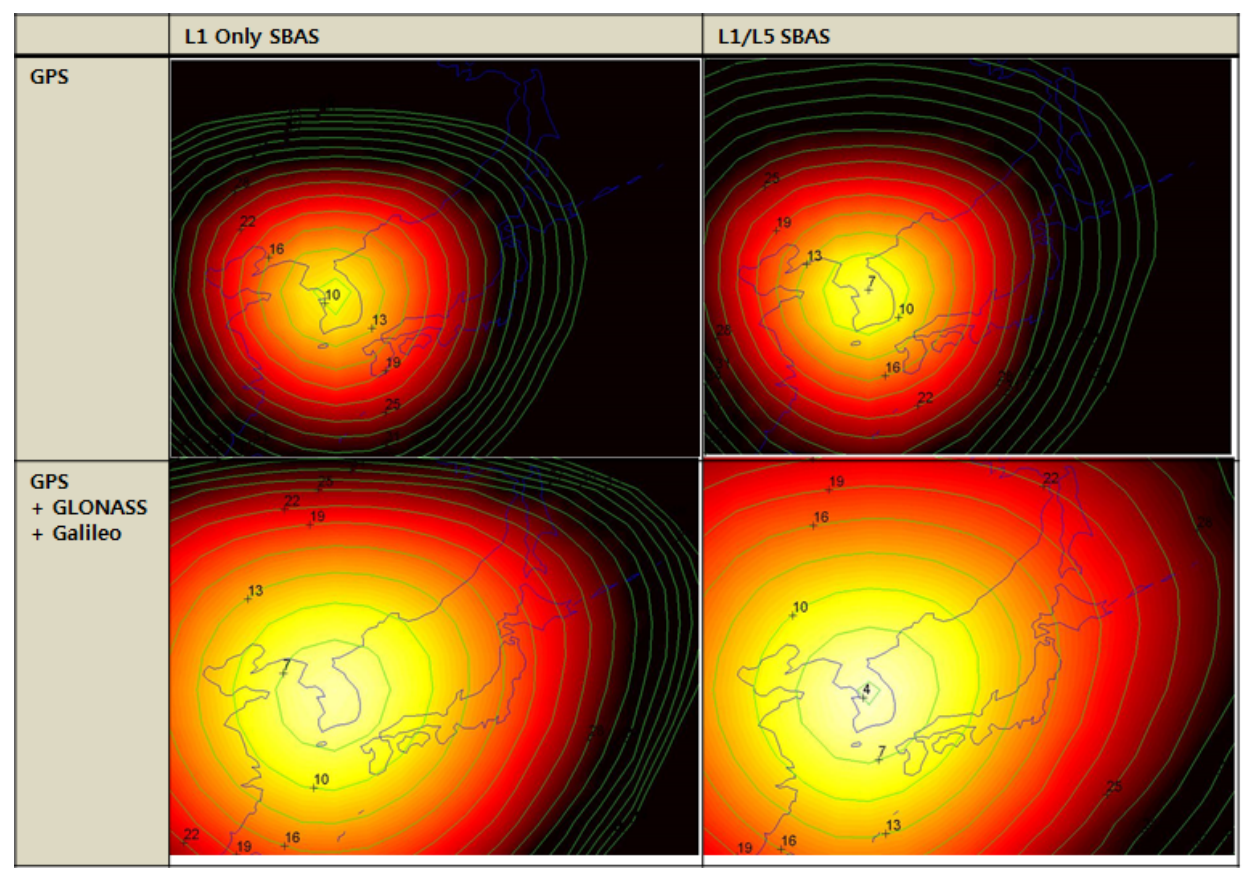

Fig. 4. User vertical protection level.

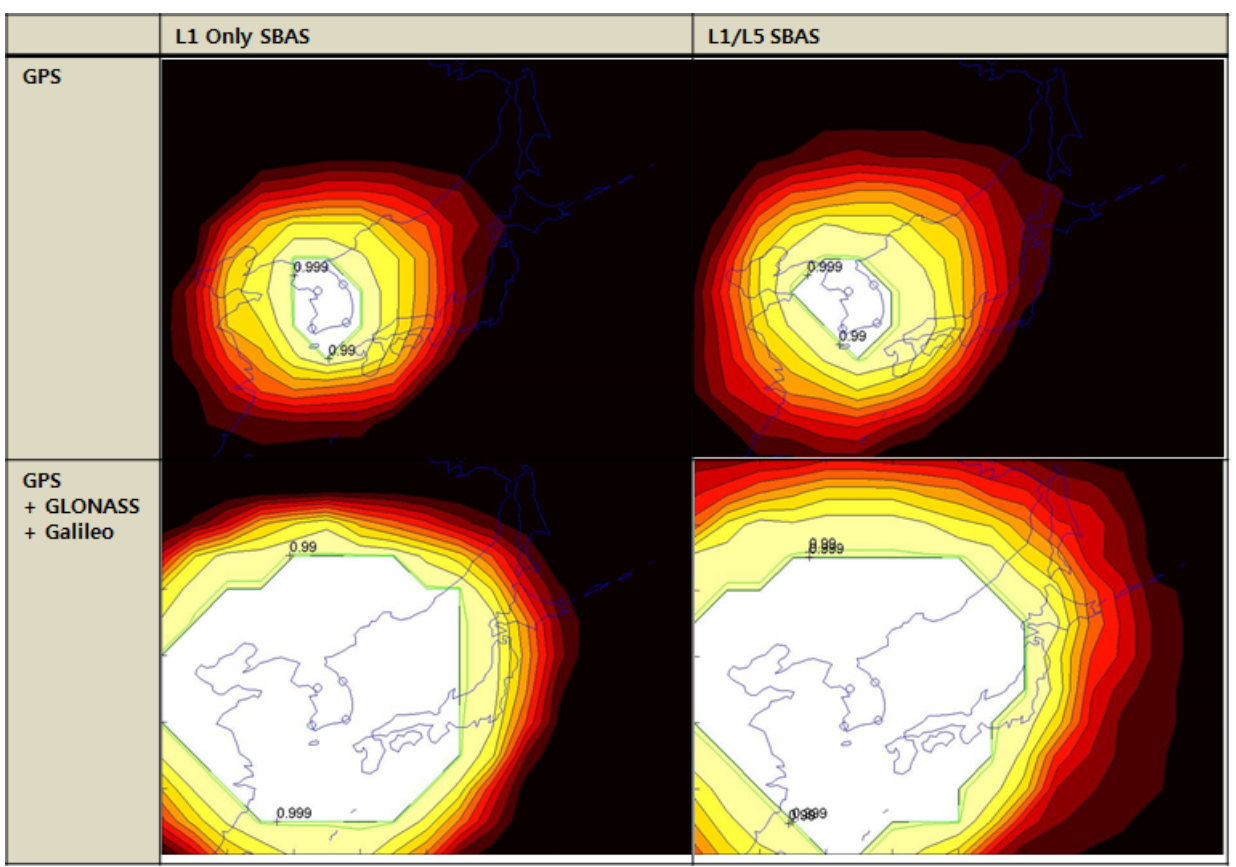

Fig. 5. LPV availability.

\section{CONCLUSIONS}

In this study, the status of the SBAS development in Korea was examined, and the performance prediction, which is necessary for the development of L1/L5 dualfrequency SBAS, was performed using simulation. Based on the results of the simulation, it is expected that dualfrequency SBAS users and single-frequency SBAS users would have similar positioning accuracy performance, but the use of dual-frequency SBAS would greatly improve the integrity and availability performance. In addition, existing single-frequency SBAS can provide complete enhancement 
information for only GPS, whereas dual-frequency SBAS can provide enhancement information for the navigation satellites of GNSS other than GPS. Therefore, resultant improvement in accuracy and availability performance is also expected.

\section{ACKNOWLEDGMENTS}

This research was supported by the Brain Korea 21 Plus Project in 2014 and the Wide Area Differential GNSS Development project of Korea funded by the Ministry of Oceans and Fisheries.

\section{REFERENCES}

GEAS, 2010, GNSS Evolutionary Architecture Study - Phase II Panel Report, available from http://www.faa.gov/ about/office_org/headquarters_offices/ato/service_ units/techops/navservices/gnss/library/documents/ media/GEASPhaseII_Final.pdf

McGraw, G. A. \& Young, Y. 2005, Dual Frequency Smoothing DGPS Performance Evaluation Studies, Proceedings of the National Technical Meeting of the ION, San Diego, CA.

RTCA SC-159, 2006, Minimum Operational Performance Standards for Global Positioning System/Wide Area Augmentation System Airborne Equipment, RTCA publication DO-229D

Schaer, S., Gurtner, W., \& Feltens, J. 1998, IONEX: The IONosphere Map Exchange Format Version 1, Proceedings of IGS AC Workshop, Darmstadt, Germany, Feb., 9-11.

Walter, T., Blanch, J., \& Enge, P. 2009, Evaluation of Signal in Space Error Bounds to Support Aviation Integrity, Proceedings of 22nd International Technical Meeting of the Institute of Navigation (ION GNSS 2009), September 22 - 25, 2009, Savannah International Convention Center, Savannah, GA.

Walter, T., Blanch, J., Phelts, R. E., \& Enge, P. 2012, Evolving WAAS to Serve L1/L5 Users, Journal of Institute of Navigation, 56, 317-327.

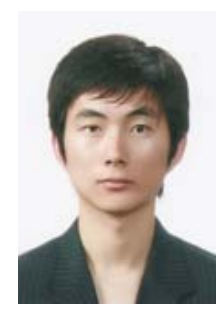

Ho Yun received the Ph.D degree in Mechanical and Aerospacce Engineering from Seoul National University in 2013. He is currently a reseach engineer in Seoul National Universiy. His research interest includes integrity monitoring, SBAS and GBAS

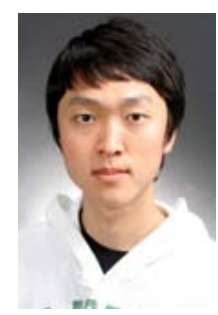

Deokhwa Han received the Bachelor degree in Mechanical and Aerospace Engineering from Seoul National University, Korea, in 2011. His research interests include WADGPS and ionosphere modeling.

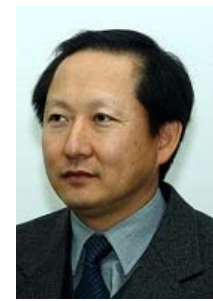

Changdon Kee received the Bachelor degree and Master degree in Mechanical and Aerospace Engineering from Seoul National University, Korea, in 1984, 1986. He received the Ph.d degree in Aeronautics and Astronautics from Stanford University, USA, in 1994 . He is currently a professor in Seoul National Universiy. His research interest includes WADGPS, CDGPS, automatic control of Unmaned vehicle, Attitude determination using GPS 